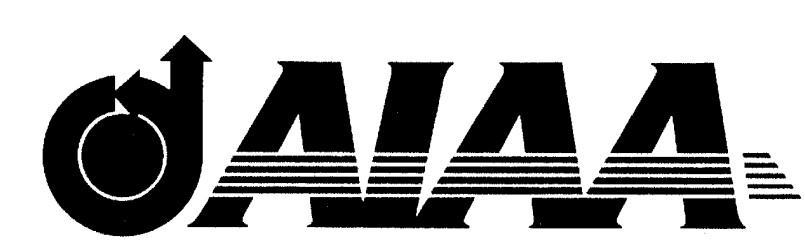

\title{
AIAA 98-2445 \\ Prediction and Validation of Mars Pathfinder Hypersonic Aerodynamic Data Base
}

Peter A. Gnoffo, Robert D. Braun, K. James Weilmuenster, Robert A. Mitcheltree, Walter C. Engelund, Richard W. Powell

NASA Langley Research Center Hampton, VA 23681-0001

\section{7th AIAA/ASME Joint Thermophysics and Heat Transfer Conference June 15-18, 1998 / Albuquerque, NM}




\title{
Prediction and Validation of Mars Pathfinder Hypersonic Aerodynamic Data Base
}

\author{
Peter A. Gnoffo, Robert D. Braun, \\ K. James Weilmuenster, Robert A. Mitcheltree, \\ Walter C. Engelund, Richard W. Powell \\ NASA Langley Research Center \\ Hampton, VA 23681-0001
}

\begin{abstract}
Postflight analysis of the Mars Pathfinder hypersonic, continuum aerodynamic data base is presented. Measured data include accelerations along the body axis and axis normal directions. Comparisons of preflight simulation and measurements show good agreement. The prediction of two static instabilities associated with movement of the sonic line from the shoulder to the nose and back was confirmed by measured normal accelerations. Reconstruction of atmospheric density during entry has an uncertainty directly proportional to the uncertainty in the predicted axial coefficient. The sensitivity of the moment coefficient to freestream density, kinetic models and center-of-gravity location are examined to provide additional consistency checks of the simulation with flight data. The atmospheric density as derived from axial coefficient and measured axial accelerations falls within the range required for sonic line shift and static stability transition as independently determined from normal accelerations.
\end{abstract}

\section{Nomenclature}

$A_{A} \quad$ axial acceleration, earth $\mathrm{g}$

$A_{N} \quad$ normal acceleration, earth g

$C_{A} \quad$ axial force coef.

$C_{D} \quad$ drag coef.

$C_{m} \quad$ pitching moment coef.

$C_{m, \alpha} \quad$ pitching moment coef. derivative, per radian

$C_{N} \quad$ normal force coef.

$D \quad$ probe diameter, $\mathrm{m}$

$h \quad$ altitude, $\mathrm{km}$

$M \quad$ Mach number

$m \quad$ entry mass, $\mathrm{kg}$

$p \quad$ pressure, $\mathrm{N} / \mathrm{m}^{2}$

$T_{\infty} \quad$ freestream temperature, $\mathrm{K}$

$S \quad$ reference area, $\mathrm{m}^{2}$

$V_{\infty} \quad$ freestream velocity, $\mathrm{m} / \mathrm{s}$

$x, y, z \quad$ Cartesian coordinates, $\mathrm{m}$

$\alpha \quad$ angle-of-attack, degrees

$\alpha_{T} \quad$ total angle-of-attack

$\left(\alpha^{2}+\beta^{2}\right)^{1 / 2}$, degrees

$\beta \quad$ angle of yaw, degrees

$\gamma \quad$ effective ratio of specific heats

$\rho_{\infty} \quad$ freestream density, $\mathrm{kg} / \mathrm{m}^{3}$

\section{Introduction}

$\mathbf{T}$ HE hypersonic aerodynamic data base used in the Mars Pathfinder entry, descent, and landing (EDL) was predominantly generated using computational fluid dynamics. While ground-based and flight data from the Viking project were used to check portions of the aerodynamic data for Pathfinder, differences in entry velocity and angle-of-attack between Pathfinder and Viking required a more critical evaluation of high temperature effects on hypersonic aerodynamics. A systematic evaluation of Pathfinder aerodynamics as a function of velocity, altitude, and angleof-attack in a continuum regime revealed two intervals during a nominal entry in which static aerodynamic instabilities would induce vehicle "wobble". The static aerodynamic instability was associated with changing sonic line location and associated pressure distributions as a function of chemical kinetics and flow energy. These predictions and explanations were published in journal articles in $1996^{1,2}$ and the "wobble" was confirmed by Pathfinder flight data (accelerometers) on July 4, 1997. The physical phenomena leading to the static aerodynamic instability are reviewed, the measured accelerations confirming the instability are discussed, and additional post-flight analyses are presented. The fidelity of comparison between pre-flight prediction and flight data significantly reduces uncertainty in the atmospheric reconstruction process and provides enhanced confidence in the application of CFD to future planetary missions with reduced design margin. 


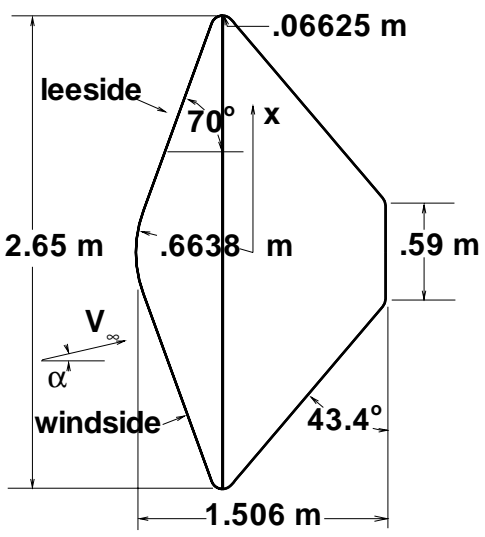

Fig. 1 Diagram of the Mars Pathfinder probe.

\section{Preflight Analysis}

The Mars Pathfinder configuration is shown in Fig. 1. The forebody is a $70^{\circ}$ half angle cone with base diameter $2.65 \mathrm{~m}$, nose radius $0.6638 \mathrm{~m}$ and shoulder radius $0.06625 \mathrm{~m}$. The on-axis reference point for aerodynamic moment coefficients is located $0.662 \mathrm{~m}$ behind the nose. The actual center-of-gravity (CG) location is $\left(x_{C G}, y_{C G}, z_{C G}\right)=(0.000107,-0.000435,0.71541)$ where $z$ is distance behind the outer mold line of the nose and $x$ and $y$ are orthogonal to the body axis.

Predictions of a static aerodynamic instability for the Mars Pathfinder vehicle associated with shifting of the sonic line from the shoulder to the nose and back have been described in the literature. ${ }^{1,2}$ Specific conclusions of the first citation relating to the occurrence of the static aerodynamic instability on the 70 degree, spherically capped Pathfinder configuration are summarized here.

(1) As the Mars Pathfinder probe descends through the Mars atmosphere the minimum value of the postshock effective $\gamma$ first decreases from frozen gas chemistry values to equilibrium values (1.094) corresponding to a velocity of $4.86 \mathrm{~km} / \mathrm{s}$. As the probe continues to decelerate through a near equilibrium post-shock gas chemistry regime, the value of $\gamma$ increases, until reaching its perfect gas value at parachute deployment $(0.42 \mathrm{~km} / \mathrm{s})$.

(2) For the spherically blunted, 70 degree half-angle cone at small angles of attack the sonic line location shifts from the shoulder to the nose cap and back again on the leeside symmetry plane because of the change in $\gamma$. This transition in sonic line location is shown in Fig. 2 for a 2 degree angle-of-attack. The subsonic flow region, as defined by the frozen sound speed, appears as the light gray region. The darker region represents zones of supersonic flow behind the bow shock. At a Mach number 22.3 on the preflight Pathfinder simulation, the sonic line on the leeside dips deep into the boundary layer over the spherical nose cap. Later in the trajectory, at Mach 16.0, a subsonic bubble forms
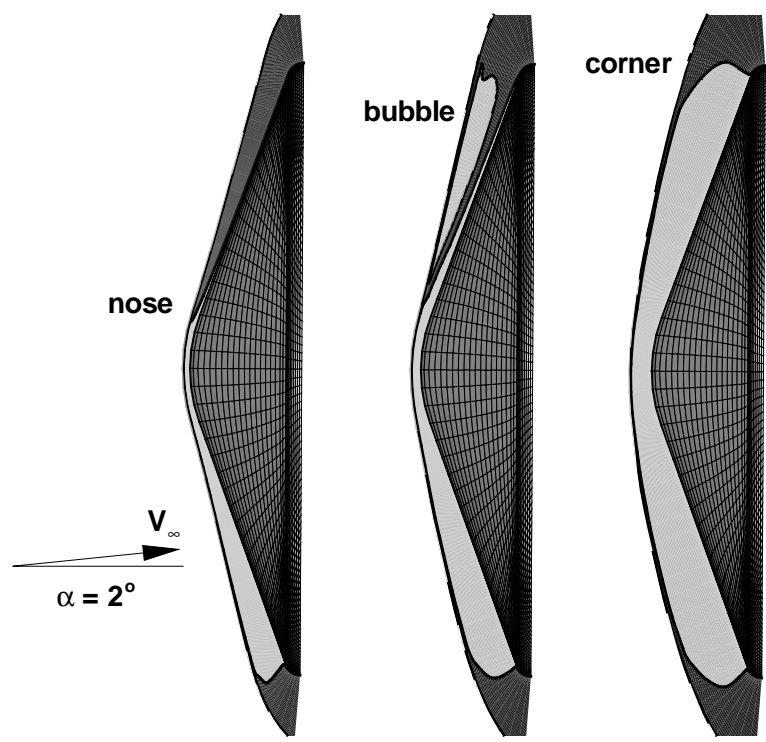

Fig. 2 Sonic line location over the Mars Pathfinder at 2 deg. angle-of-attack and Mach numbers equal to 22.3 (left), 16.0 (center), and 9.4 (right) showing the effect of gas chemistry.

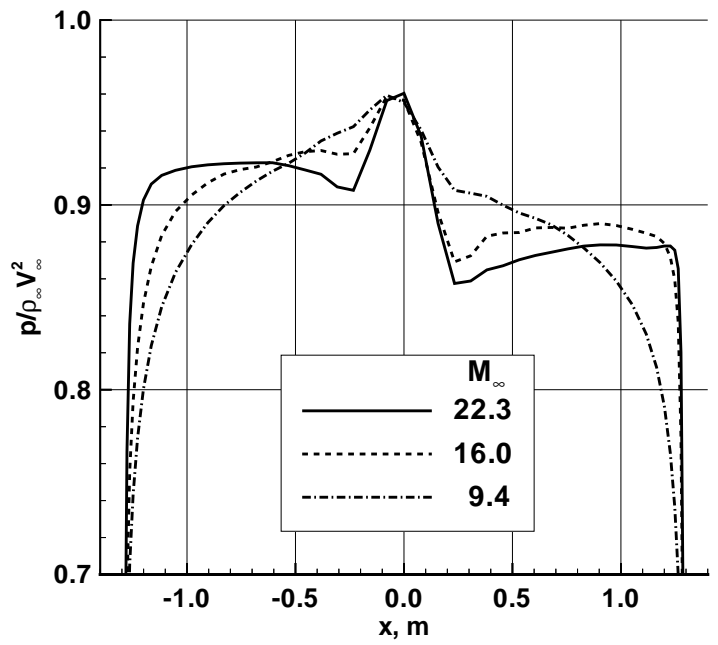

Fig. 3 Pressure distributions in the plane of symmetry over the Mars Pathfinder at 2 deg. angle-ofattack and Mach numbers equal to 22.3, 16.0, and 9.4 showing the effect of gas chemistry.

immediately behind the bow shock just downstream of an inflection point in the bow shock. The sonic line from the nose skirts above the edge of the boundary layer. Still later, at Mach 9.4, the bubble expands to merge with the subsonic region at the boundary layer edge. The inflection point on the bow shock disappears.

(3) Pressure distributions on the cone fustrum approaching the shoulder tend to be very flat when the sonic line sits forward over the spherical nose, as shown in Fig. 3 for Mach 22.3. Effects of the expansion over the shoulder can only be communicated upstream 
through the subsonic portion of the boundary layer. In contrast, pressure distributions on the cone fustrum approaching the shoulder tend to be more rounded when the sonic line sits on the shoulder, exhibiting a more pronounced influence of the expansion on the upstream flow as shown in Fig. 3 for Mach 9.4.

(4) In general, windside pressures exceed leeside pressures on the cone fustrum, producing a stabilizing moment that pitches the probe back to zero angle-of-attack. However, the behavior of the pressure distribution in the vicinity of the shoulder significantly influences the pitching moment coefficient because of the larger moment arms and surface area at the edge of the probe as compared to the inboard nose and fustrum regions. For the Mars Pathfinder probe at 2 deg. angle-of-attack, the flat, leeside pressures approaching the shoulder (when the sonic line sits over the nose) can exceed the rounded windside pressures approaching the shoulder (when the sonic line sits over the shoulder). The net effect of this crossover distribution near the shoulder tends to pitch the probe to higher angles of attack (static instability). The overall balance (crossover-point location) is sensitive to freestream conditions and corresponding gas chemistry within the shock layer. The combination of small angle-of-attack, large cone angle, windside sonic line over the shoulder, and leeside sonic line over the nose with a secondary, subsonic zone above the leeside fustrum yield conditions which favor (but do not necessarily guarantee) a destabilizing pitching moment.

(5) Representative parts of the aerodynamic data base as a function of velocity during the hypersonic, continuum portion of the trajectory are shown in Fig. 4. Conditions for a positive, destabilizing moment coefficient derivative $C_{m, \alpha}$ occur twice in the Mars Pathfinder mission. $\left(C_{m}\right.$ is zero when $\alpha_{T}$ equals zero; $C_{m, \alpha}$ is positive where the $C_{m}$ curve for $\alpha=2 \mathrm{deg}$. crosses the $C_{m}=0$ axis in Fig. 4 b.) The first occurrence $\left(7.5>V_{\infty}>6.5 \mathrm{~km} / \mathrm{s}, 51>h>37 \mathrm{~km}\right.$, vicinity of peak heating for this trajectory) results from the transition in the sonic line location as a function of gas chemistry changing from nonequilibrium to equilibrium. The second occurrence $\left(4.0>V_{\infty}>3.1\right.$ $\mathrm{km} / \mathrm{s}, 25>h>22 \mathrm{~km}$ ) results from the transition in the sonic line location as a function of decreasing flow enthalpy in a near equilibrium gas chemistry regime.

\section{Measured Accelerations}

Measured accelerations during Pathfinder's entry, descent, and landing (EDL) validate these earlier conclusions. The raw, three-axis accelerometer data for the hypersonic portion of EDL are presented in Fig. 5. In this figure, the $\mathrm{z}$-axis is in the axial direction. The $\mathrm{x}$ - and $\mathrm{y}$ - axes are fixed on the body as it spins (approximately $2 \mathrm{rpm}$ ), pitches and precesses during descent. Peak dynamic pressure occurs approximately 78 seconds after entry interface. In the vicinity of peak

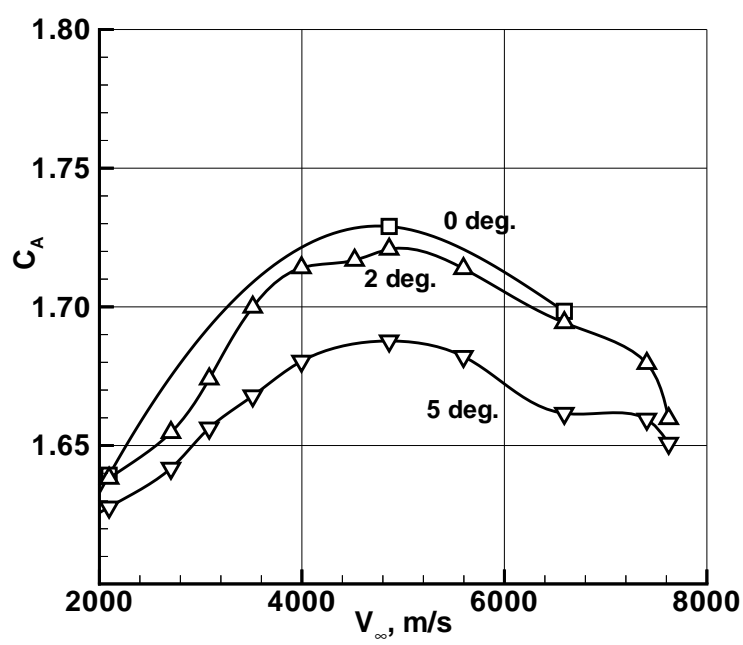

a) Axial coefficient

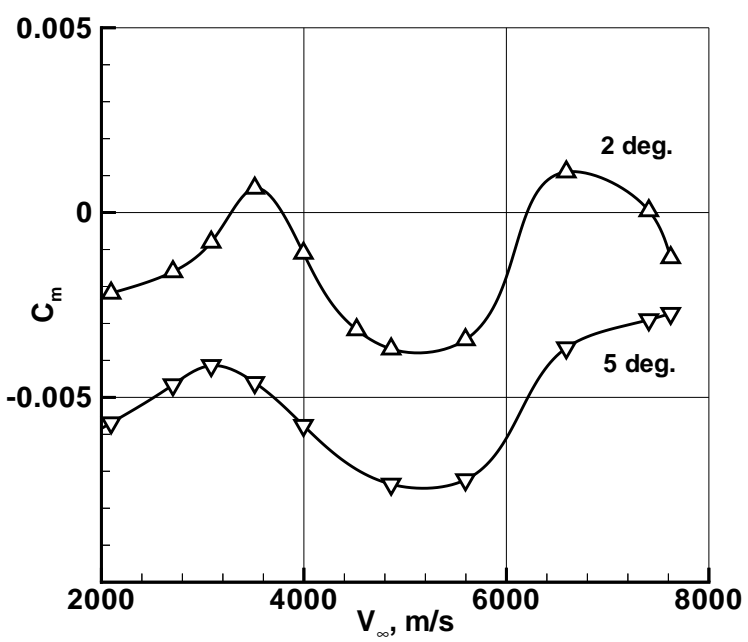

b) Pitching moment coefficient

Fig. 4 Predicted aerodynamic coefficients as function of velocity and angle-of-attack for early Mars Pathfinder trajectory with ballistic coefficient equal to 45 .

dynamic pressure, the magnitude of the off-axis accelerations abruptly decrease, corresponding to a brief period where CFD simulations show the sonic line fixed to the spherical nose at small $\alpha_{T}$.

The trend is more clearly observed in Fig. 6 in which a total normal acceleration $\left(A_{N}=\left(A_{x}^{2}+A_{y}^{2}\right)^{1 / 2}\right)$ is plotted as a function of time. In this figure, a rather abrupt increase in normal acceleration is observed on either side of the relative low level $\left(A_{N} \approx 0.014 \mathrm{~g}\right)$ near peak dynamic pressure to a relatively high level ( $A_{N} \approx 0.04 \mathrm{~g}$ ). This change closely corresponds to the predicted times when the sonic line shifts from the shoulder to the nose $(\approx 71 s)$ and then back again to the shoulder $(\approx 82 s)$. 


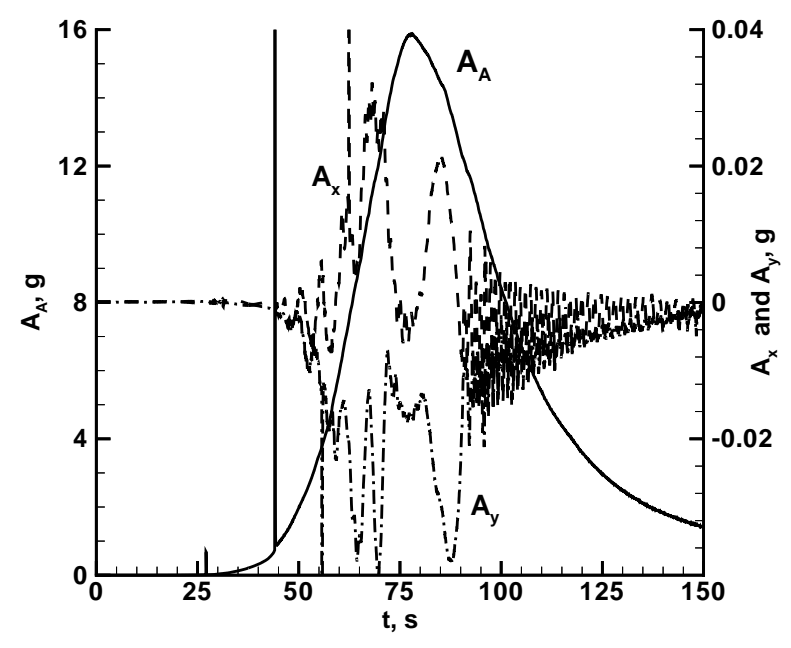

Fig. 5 Science accelerometer measurements recorded on Mars Pathfinder during 2.5 minutes following entry interface with the Mars atmosphere.

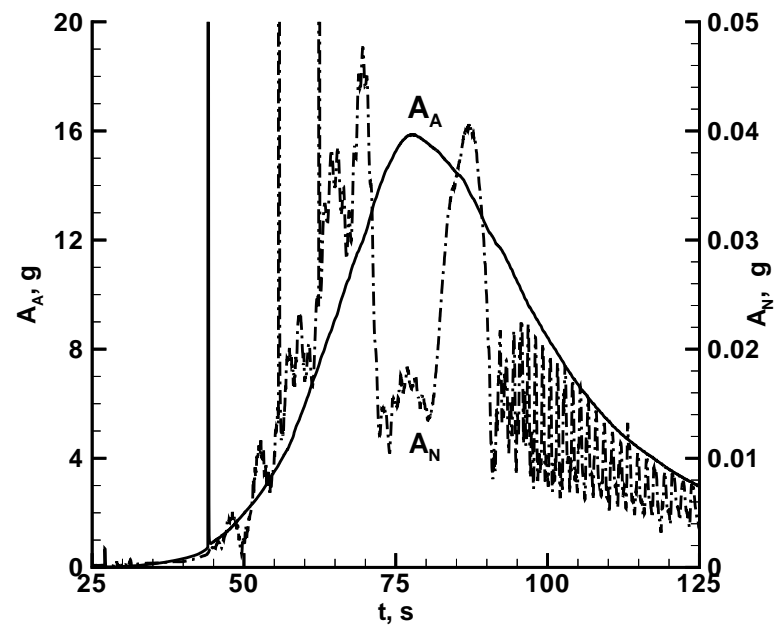

Fig. 6 Total off-axis acceleration recorded during 100 second interval surrounding peak dynamic pressure.

In general, the correspondence of measured accelerations and predicted aerodynamic coefficients is complicated by the unknown freestream density. Velocity can be derived from integrated accelerations but density is not directly measured. This complicating factor can be removed by examining the ratio of normal to axial forces and normal to axial aerodynamic coefficients.

$$
\frac{C_{N}}{C_{A}}=\frac{\frac{F_{N}}{1 / 2 \rho_{\infty} V_{\infty}^{2} S}}{\frac{F_{A}}{1 / 2 \rho_{\infty} V_{\infty}^{2} S}}=\frac{m A_{N}}{m A_{A}}=\frac{A_{N}}{A_{A}}
$$

The predicted values of normal to axial force coefficient using the initial state vector determined four hours prior to entry interface in the six-degree-of- freedom (6-DOF) Program to Optimize Simulated Trajectories (POST) ${ }^{3}$ code and aerodynamic coefficient tables derived from CFD simulations ${ }^{1}$ are presented in Fig. 7a. The corresponding measured values of normal to axial accelerations are presented in Fig. 7b. The predicted and measured results show very good qualitative and quantitative agreement. For example, the effects of the predicted and measured static aerodynamic instability between 82 and 90 seconds are evident in both the magnitude and duration of the pulse. The time averaged aerodynamic coefficient ratio in the vicinty of peak dynamic pressure between 71 and 82 seconds is also in good agreement with magnitude and duration of the measured ratio of accelerations when the sonic line sits over the spherical nose. The predictions show a much larger oscillation about the mean in this domain than was measured. An overly conservative specification for $C_{m, q}$ in this region in the simulation is the suspected cause. The magnitude and frequency of oscillations beyond 90 seconds are in fair agreement but the mean value of measured acceleration ratio (.0016) remains at a constant level while the mean value of the simulated acceleration ratio starts lower (.0008) and gradually rises to .0024. Reasons for these discrepancies are not yet understood.

The total angle-of-attack $\alpha_{T}$ predicted in the 6-DOF POST analysis is presented in Fig. 8a. The corresponding values in flight (Fig. $8 \mathrm{~b}$ ) are derived from aerodynamic tables of $C_{N} / C_{A}$ as a function of $\alpha_{T}$ using the measured ratio of normal to axial accelerations. Here again, both qualitative and quantitative agreement are generally good and serve to validate the simulation methodology for aerodynamics.

\section{Atmospheric Reconstruction}

Reconstruction of the Mars atmosphere based on measured accelerations on Mars Pathfinder has been described by Spencer et. al. ${ }^{4}$ Velocities are derived from measured accelerations. Densities are derived from measured axial accelerations, the derived velocities, and the predicted aerodynamic data base for $C_{A}$ as a function of velocity, Mach number, and total angle-of-attack. The point-by-point transformation is given by

$$
\rho_{\infty}=\frac{2 A_{A}}{V_{\infty}^{2} C_{A}\left(\frac{S}{m}\right)}
$$

Errors in the predicted aerodynamic data base are linearly related to errors in the derived densities. The reconstructed density is plotted as a function of velocity in Fig. 9. The off-axis accelerations are plotted in this same figure to show the correspondence of simulation coordinates (velocity, density) with the onset and decay of the aerodynamic instabilities at small angles of attack. 


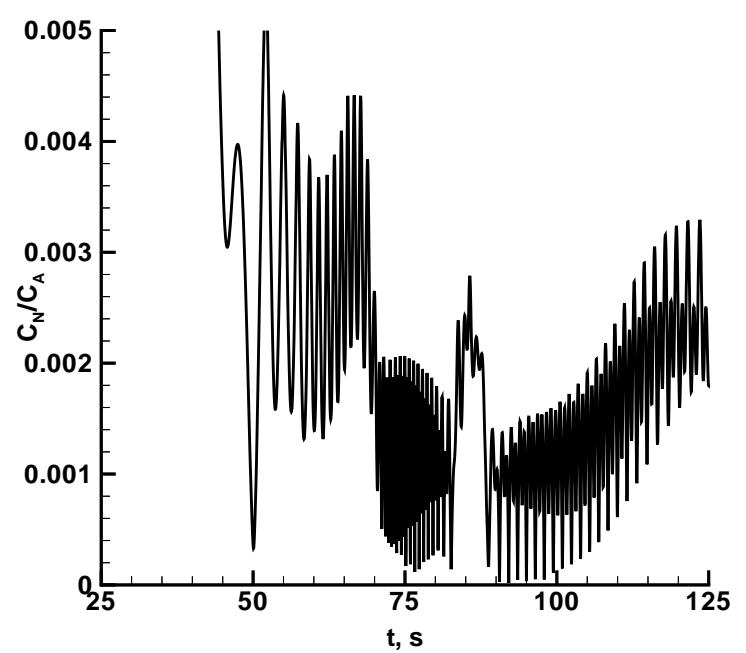

a) Predicted ratio of normal to axial force coefficients from 6-DOF POST

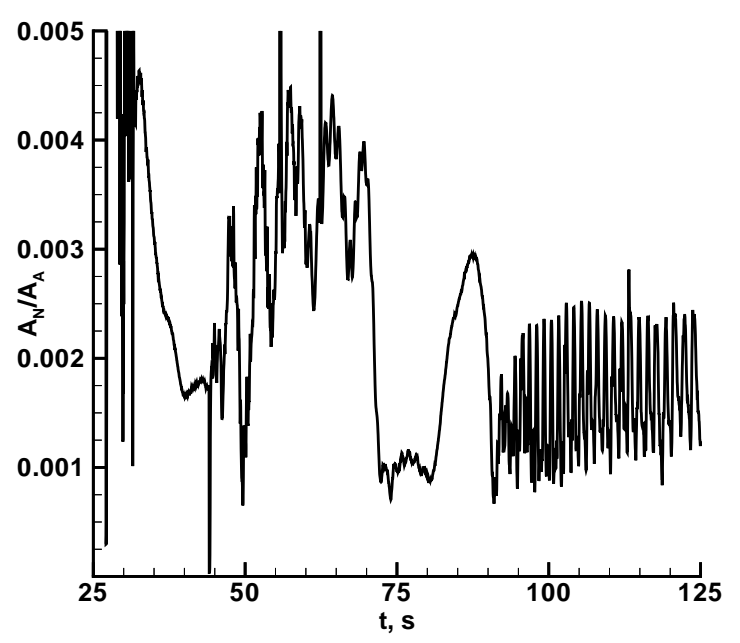

b) Measured normal to axial acceleration ratio

Fig. 7 Ratio of normal acceleration to axial acceleration recorded during 100 second interval surrounding peak dynamic pressure. The ratio is equivalent to the value of $C_{N} / C_{A}$ for Pathfinder at its total angle-of-attack $\alpha_{T}$.

\section{Uncertainty Analysis}

The uncertainty in center-of-gravity (CG) location is $\pm 5 \mathrm{~mm}$ in the axial and $\pm 1 \mathrm{~mm}$ in the radial directions. Given $C_{A} \approx 1.7$ over the hypersonic, continuum portion of the trajectory, the uncertainty in $C_{m}$ associated with the uncertainty of the CG location in the pitch plane is $\Delta C_{m}=C_{A} \Delta r / D= \pm 0.00064$. The uncertainty in $C_{m}$ associated with an axial translation of the CG is two orders of magnitude smaller for small angles of attack. Note that there is no uncertainty in the aerodynamic reference point; we simply treat $\mathrm{CG}$ location uncertainty as equivalent to a corresponding

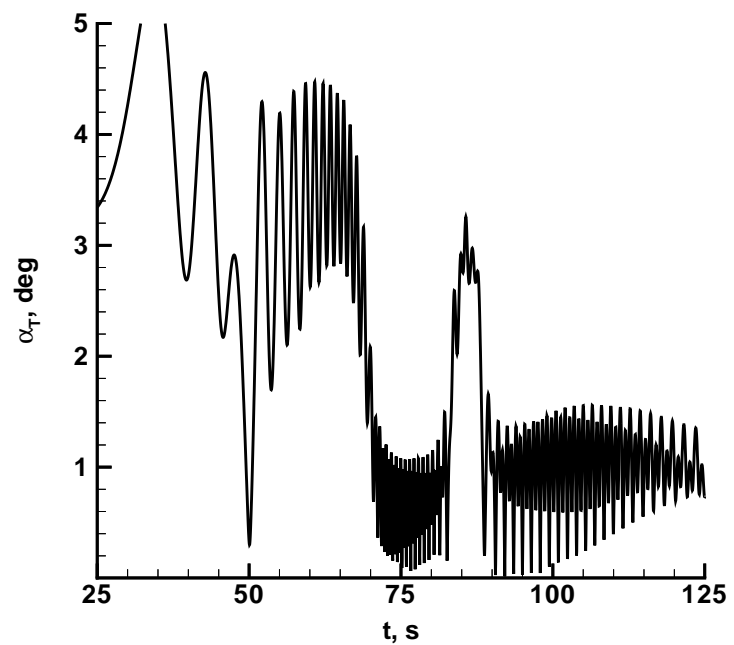

a) Predicted $\alpha_{T}$ from 6-DOF POST

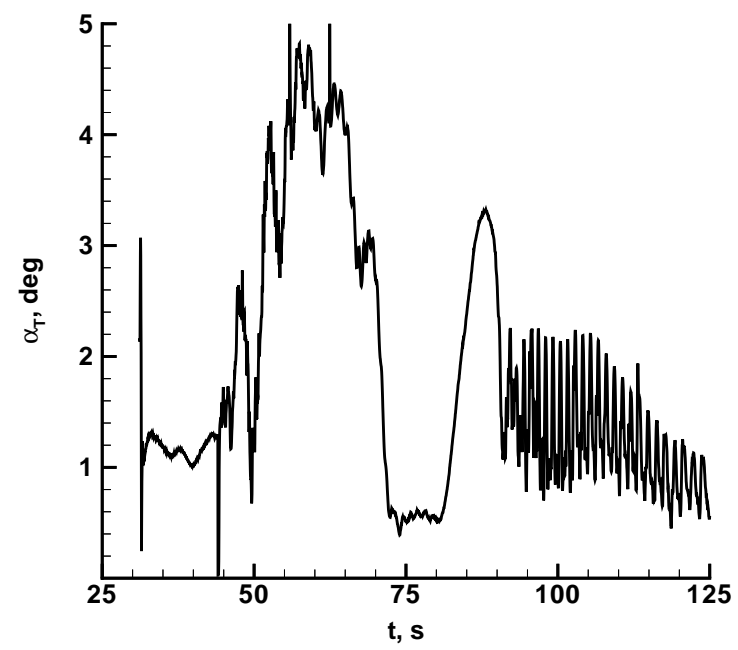

b) Derived $\alpha_{T}$ from recorded accelerations

Fig. 8 Comparison of total angle-of-attack $\alpha_{T}$ predicted by 6-DOF POST using initial state vector determined 4 hours prior to entry interface with values derived from measured accelerations.

change in the reference location.

All of the solutions generated for the next section and most of the solutions used to generate the aerodynamic data base were obtained on a surface grid of 30 by 60 cells (half body to symmetry plane) with 64 cell normal to the body. A grid refinement to 40 by 80 cells for a case with $V_{\infty}=3515 \mathrm{~m} / \mathrm{s}$ at $\alpha=2 \mathrm{deg}$ yielded a change in $C_{N} / C_{A}$ of less than 0.00015 , so that the predicted ratio of normal to axial acceleration has an uncertainty of at least \pm 0.00015 . The change in $C_{m}$ for this refinement was less than 0.0004 . In a related grid coarsening test the solution at the $6000 \mathrm{~m} / \mathrm{s}$ and $\alpha=2$ deg. was computed on a grid with every other mesh point removed in all three computational direc- 


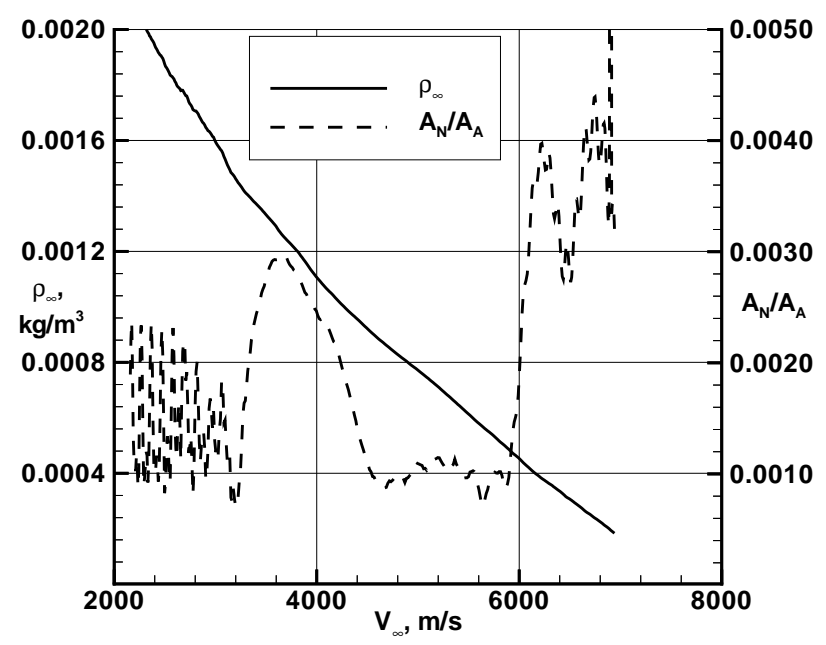

Fig. 9 Reconstructed density and ratio of measured accelerations as function of velocity for Mars Pathfinder during flight through the continuum, hypersonic flow regime.

tions. This test yielded a change in $C_{N} / C_{A}$ of less than 0.00012 and a change in $C_{m}$ less than 0.0003 .

Measured axial accelerations in the hypersonic, continuum domain are accurate to within $1 \%$. Uncertainties in measured normal accelerations are more difficult to characterize; they are expected to be less than $1 \%$ of full scale $\left(\Delta A_{N}<.008 \mathrm{~g}\right)$ in this domain. Velocities are derived by integrating the measured accelerations in time from the initial state vector. Uncertainties in the derived velocities should be less than $1 \%$ because random errors in measured accelerations smooth out and calibration checks remove bias errors in measured accelerations. Furthermore, an independent check of derived velocity with altimeter data prior to parachute deployment showed differences less than $1 \%$.

Uncertainties in the derived density are linearly related to the total uncertainty in the simulated values $C_{A}$ which include uncertainties associated with $\alpha_{T}$ and gas chemistry model. (Recall Eq. 2.) Uncertainties in the derived density are quadratically related to the total uncertainty in freestream velocity. Comparison of LAURA simulations to ground based experimental data for an axisymmetric blunt body (METEOR ${ }^{5}$ ) at Mach 6 in which uncertainties in gas chemistry (perfect gas) and $\alpha_{T}$ (sting mounted) are near zero (relative to the flight case) show differences less than $2 \%$ in $C_{A}$, and less than $5 \%$ in $C_{N}$ and $C_{m}$. Uncertainty in $C_{A}$ associated with the gas model is difficult to define, but thought to be approximately $1 \%$ for low angles of attack based on computational analysis in the next section. Uncertainty in the derived $\alpha_{T}$ is implicitly related to the uncertainty in $C_{N}$ and measured accelerations; and, given the values discussed above and the tables and figures from the preflight analysis, ${ }^{1}$ the un- certainty in $\alpha_{T}$ should be less than 0.6 degrees. The uncertainty in $C_{A}$ due to the uncertainty in $\alpha_{T}$ is less than $0.7 \%$. Effects of base pressure on the hypersonic aerodynamic drag are less than $0.5 \% .^{1}$ Consequently, the total RMS value for uncertainty in $C_{A}$ in the hypersonic, continuum domain at low angles of attack is less than $3 \%$. The corresponding total RMS value for uncertainty in $\rho_{\infty}$ in this domain is less than $4 \%$. Comparison of computed drag coefficient ${ }^{1}$ to experimental data ${ }^{6}$ for the Viking configuration in ballistic range tests in $\mathrm{CO}_{2}$ fall within this $3 \%$ estimate.

As a final note, all of the simulations assume laminar flow. The assumption should be valid for most of the hypersonic, continuum trajectory presented here. At the $V_{\infty}=3996 \mathrm{~m} / \mathrm{s}$ trajectory point $\left(M_{\infty}=18\right.$, $R e_{D, \infty}=1.2$ million), the value of $R e_{\theta} / M_{e}$ is less than 500 over most of the cone and drops below zero as flow expands over the shoulder. In recent comparisons to engineering code assessments of $R_{e} / M_{e}$ the CFD valuation of this quantity is roughly twice the engineering code valuation. Assuming a transition criteria of $R e_{\theta} / M_{e}=250$ for blunt bodies from engineering code assessments turbulent flow may occur near this condition. The contribution of laminar skin friction to $C_{A}$ is less than $0.2 \%$ as noted in block 2 of Table $4\left(M_{\infty}=16, V_{\infty}=3515 \mathrm{~m} / \mathrm{s}, \alpha=2\right.$ deg. $)$ in the preflight analysis. ${ }^{1}$ If we assume: (1) no relaminarization at the shoulder where the strongest contribution of shear to $C_{A}$ will occur; (2) negligible displacement thickness effects of a turbulent boundary layer on pressure for this very blunt configuration; and (3) a factor 5 increase in shear over laminar values, then an additional uncertainty of $1 \%$ in $C_{A}$ is introduced. The contribution from turbulent shear at the shoulder to $C_{m}$ at small angles of attack is more difficult to estimate because of the greater importance of differences between windside shoulder and leeside shoulder contributions. The laminar shear contribution to $C_{m}$ for the $M_{\infty}=16$ simulation noted above is a destabilizing increment equal to 0.00012. Again assuming a factor 5 increase in the turbulent contribution leads to an additional uncertainty in $C_{m}$ equal to 0.0006 .

\section{Sensitivity Analysis}

The aerodynamic data base used in the POST code was originally generated on the basis of a simulated trajectory for a $418 \mathrm{~kg}$ vehicle with a ballistic coefficient of $45 \mathrm{~kg} / \mathrm{m}^{2}$ in the Mars-GRAM atmospheric model. ${ }^{7}$ The vehicle grew as the design matured and the actual entry mass was $585.3 \mathrm{~kg}$ with a ballistic coefficient of $62 \mathrm{~kg} / \mathrm{m}^{2}$. In the final design phase, cruise and entry operations, and post-flight analysis, the Clancy atmospheric model ${ }^{8,9}$ was used. The POST code used velocity as a curve fit parameter for aerodynamic coefficients above Mach 12 in the continuum regime because of the important role of effective $\gamma$ on aerodynamics and its dependence on total enthalpy. 
Below Mach 8, Mach number was used as a correlating parameter to emphasize compressibility effects on aerodynamics. Between Mach 8 and 12, a bridging function is used. As a result of differences in mass, entry angle, and atmospheric model, a comparison of the early preflight and post-flight trajectories will yield different densities for the same velocity. As a point of reference, the data base used aerodynamic coefficients simulated at $V_{\infty}=3996 \mathrm{~m} / \mathrm{s}$ and $\rho_{\infty}=1.5510^{-3}$ $\mathrm{kg} / \mathrm{m}^{3}$; the density at that same velocity in the reconstructed atmosphere was $\rho_{\infty}=1.1310^{-3} \mathrm{~kg} / \mathrm{m}^{3}$.

In the continuum, hypersonic flight regime, the change in density would be expected to influence aerodynamic coefficients through Reynolds number and gas kinetics. As discussed in the previous section, the influence of Reynolds number is expected to be small, but the influence of gas kinetics on the effective $\gamma$ is unknown. The primary purpose of this section is to quantify the sensitivity of the aerodynamic data base to perturbations in density and the gas kinetic model. A secondary purpose of this section is to critically evaluate the transition regions between statically stable and unstable regions and check that the simulation is consistent with measured normal accelerations.

The moment coefficient at $\alpha=2 \mathrm{deg}$. for the postflight, simulated trajectory is plotted over the simulated Pathfinder measurements of normal to axial acceleration as a function of velocity in Fig. 10. The value of $C_{m}$ at $\alpha=2 \mathrm{deg}$, designated $C_{m}(2)$, is proportional to $C_{m, \alpha}$ at $\alpha=0$ assuming a forward difference approximation $C_{m, \alpha}(0)=\left(C_{m}(2)-0\right) * 90 / \pi$. The purpose of this plot is to identify values of $C_{m}(2)$ that are associated with the transition between regions of static instability and static stability. The transition region is delineated with the rectangles.

We introduce an assumption that critical values in $C_{m}(2)$ may be defined that serve to delineate the various stability regions as determined from measured accelerations. In each transition region an upper limit on $C_{m}(2), C_{m, U}$, is identified such that any simulation with $C_{m}(2)>C_{m, U}$ would be expected to be in a region of static instability. In like manner, a lower limit on $C_{m}(2), C_{m, L}$, is identified such that any simulation with $C_{m}(2)<C_{m, L}$ would be expected to be in a region of static stability. If $C_{m, U}>C_{m}(2)>C_{m, L}$ one would expect measured accelerations to be within the transition between the two stability regions.

Values of $C_{m, U}$ and $C_{m, L}$ are obtained from analysis of four different simulations of the Pathfinder trajectory through the reconstructed atmosphere. The vehicle was numerically flown using the 6-DOF POST code with the baseline aerodynamic data base and three perturbations in which the curve fits of aerodynamic coefficients as a function of velocity were shifted by $+1 \%,-5 \%$, and $-10 \%$ in velocity space (i.e. $\left.C_{x}=C_{x}\left(V_{\infty}-0.1 V_{\infty}\right)\right)$. This shift causes the instabilities to arise earlier or later in the simulated trajectory

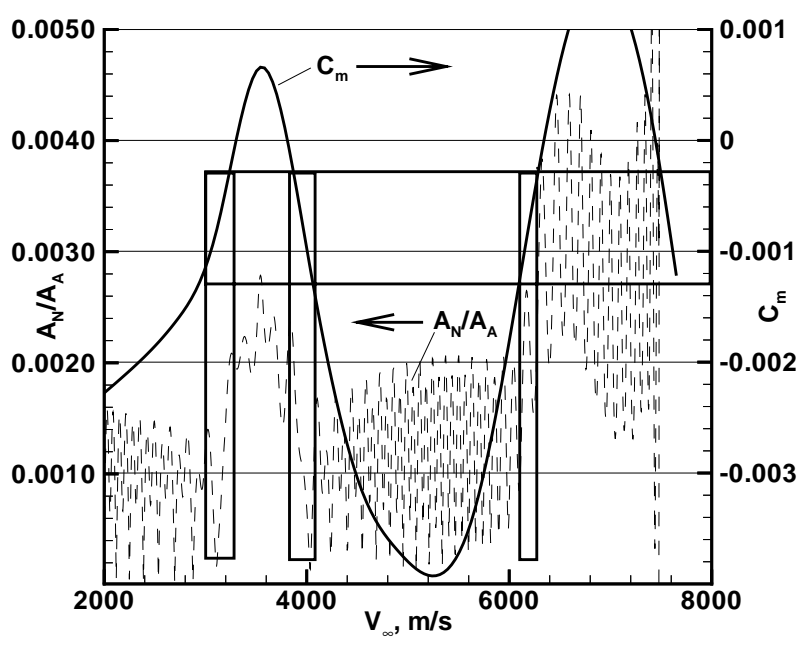

Fig. 10 Ratio of normal to axial accelerations and moment coefficient at $\alpha=2$ deg. for the preflight simulated trajectory as a function of velocity. The transition between regions of static instability and static stability are bounded with the rectangular boxes.

and allows us to check the assumption that critical values for $C_{m, U}$ and $C_{m, L}$ can be defined. Values for $C_{m, U}$ and $C_{m, L}$ based on this subjective selection criterion are respectively -0.0003 and -0.0013 (see bounding boxes in Fig. 10). When the major contributor to uncertainty in $C_{m}$, CG location, is included the transition range expands to +0.00034 to -0.00194 .

\section{Results and Discussion}

Four test points are chosen along the reconstructed trajectory and are identified in Table 1 and in Fig. 11. The simulated data in Fig. 11 is based on flight through the reconstructed atmosphere, in contrast to the data in Fig. 7 a) based on flight through the preflight atmospheric model. Except for the lack of high frequency oscillations in the measured data between $3400 \mathrm{~m} / \mathrm{s}$ and $6000 \mathrm{~m} / \mathrm{s}$ there is fairly good qualitative agreement between the measured and simulated data. (Temporal resolution of the accelerometers should have picked up such oscillations if they were present.) The magnitude of measured accelerations is slightly larger (15-30\%) than the temporally averaged level of simulations in the statically stable region between 4500 and $6000 \mathrm{~m} / \mathrm{s}$ and in the statically unstable region between 3400 and $4000 \mathrm{~m} / \mathrm{s}$. The measured duration of the statically stable region in velocity space is shorter than the simulated duration. These observations are consistent with trends shown in Fig. 9 of Braun et. al. ${ }^{2}$ in which the $\mathrm{CG}$ moves off the axis. The current simulation assumes a $0.448 \mathrm{~mm}$ offset CG location. 


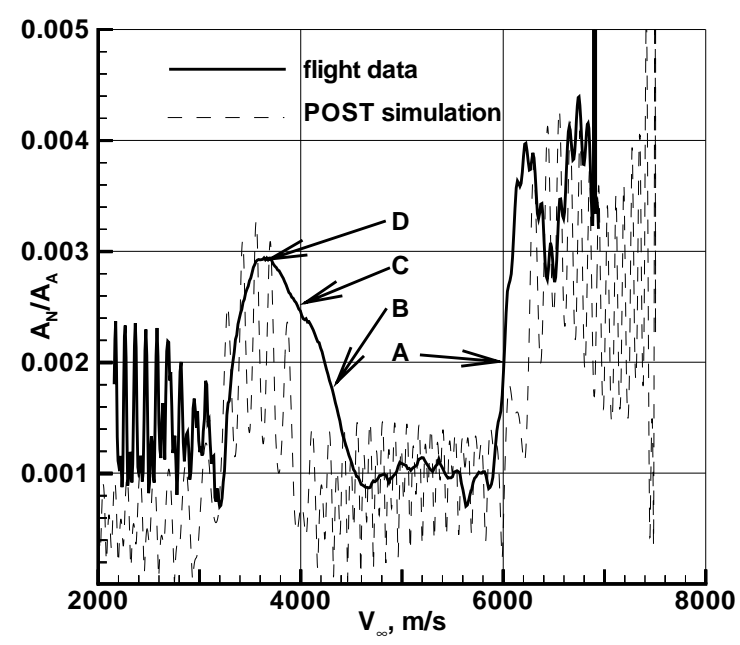

Fig. 11 Comparison of $A_{N} / A_{A}$ as measured in flight and as simulated with the POST code in the reconstructed atmosphere using the CFD derived data base for aerodynamic coefficients.

Table 1 - Test Points and Baseline Aerodynamics

\begin{tabular}{|c|c|c|c|c|c|}
\hline Pt. & $V_{\infty} \frac{\mathrm{m}}{\mathrm{s}}$ & $\rho_{\infty}, \frac{\mathrm{kg}}{\mathrm{m}^{3}}$ & $C_{A}(2)$ & $C_{N}(2)$ & \\
\hline $\bar{A}$ & 6000 & & 1.707 & & \\
\hline B & & & & & \\
\hline $\mathrm{C}$ & & & & & -0. \\
\hline & & & 1.698 & 0.001883 & +0.00033 \\
\hline
\end{tabular}

The aerodynamic coefficients for the baseline eight species $\left(\mathrm{CO}_{2}, \mathrm{CO}, \mathrm{N}_{2}, \mathrm{O}_{2}, \mathrm{NO}, \mathrm{C}, \mathrm{N}, \mathrm{O}\right)$ kinetic model $^{10}$ are included in the Table 1. Point $\mathrm{A}$ is in the middle of the transition region, near peak heating and prior to peak dynamic pressure where the sonic line is completing its movement to the nose with decreasing $\gamma$. Point B is in the middle of the next transition, after peak dynamic pressure in which the sonic line is beginning to move back to the shoulder from the nose with increasing $\gamma$ associated with decreasing flow enthalpy. At this point in Fig. 11 the POST simulation indicates a region of static stability, in apparent disagreement with the flight data. Point $\mathrm{C}$ is at the end of this transition region containing Point $\mathrm{B}$; the velocity here corresponds to one used in establishing the aerodynamic data base. Point $\mathrm{D}$ is at the local maximum for $A_{N} / A_{A}$.

Given the assumptions stated previously, values of $C_{m}(2)$ for Points A-C would be expected to be between $C_{m, U}$ and $C_{m, L}$. The value of $C_{m}(2)$ for Point $\mathrm{D}$ would be expected to be greater than $C_{m, U}$. As is evident in Table 1, the baseline kinetic model yields values of $C_{m}(2)$ which are consistent with the critical values of $C_{m, U}$ and $C_{m, L}$ for points $\mathrm{A}, \mathrm{C}$, and $\mathrm{D}$, even without adding the uncertainty bar associated with CG location. The uncertainty bar around point B associated with CG location just overlaps the lower limit

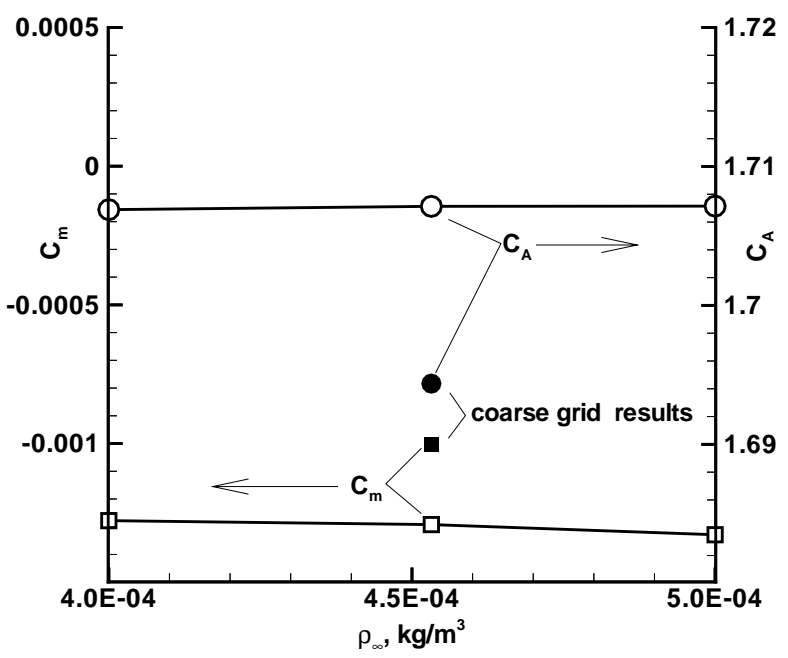

Fig. 12 The computed sensitivity of Pathfinder aerodynamic coefficients on freestream density for a velocity of $6000 . \mathrm{km} / \mathrm{s}$.

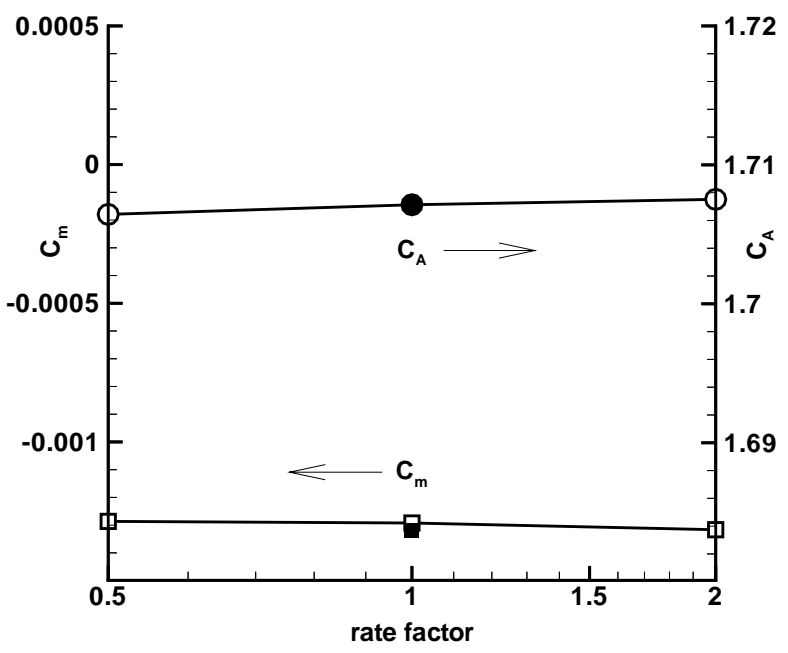

Fig. 13 The computed sensitivity of Pathfinder aerodynamic coefficients on dissociation rate coefficient for $\mathrm{CO}_{2}$ at a freestream density 0.0004532 $\mathrm{kg} / \mathrm{m}^{3}$ and velocity of $6000 . \mathrm{km} / \mathrm{s}$.

for $C_{m, L}$ that defines the transition region.

The sensitivity of $C_{A}(2)$ and $C_{m}(2)$ with respect to $\rho_{\infty}$ and kinetic model at point $\mathrm{A}$ are shown respectively in Figs. 12 and 13. Open symbols represent the baseline grid results $(60 \times 30 \times 64)$ and the filled symbols show a result with a factor two coarsening in all coordinate directions. Aerodynamic coefficients are insensitive to perturbations as large as $10 \%$ about the nominal density of $4.5310^{-4} \mathrm{~kg} / \mathrm{m}^{3}$. The value for $C_{m}(2)$ at Point $\mathrm{A}$ is at the lower edge of the expected transition range. Aerodynamic coefficients are also insensitive to changes in the dissociation rate constant for $\mathrm{CO}_{2}$ by factors varying from 0.5 to 2.0 . 


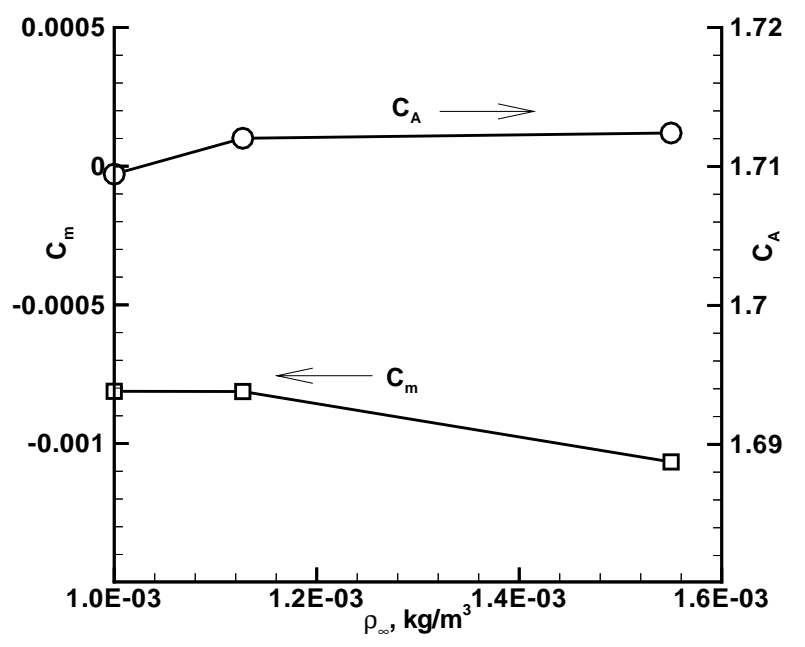

Fig. 14 The computed sensitivity of Pathfinder aerodynamic coefficients on freestream density for a velocity of $3996 . \mathrm{km} / \mathrm{s}$.

Inspection of $\mathrm{CO}_{2}$ mass fraction in all three solutions shows that $\mathrm{CO}_{2}$ is substantially depleted, other species play a more dominant role in establishing $\gamma$. Changing the effective temperature for dissociation in all such reactions at Point $\mathrm{A}$ from the baseline model ( $T_{\text {eff }}=T^{0.5} T_{V}^{0.5}$, open symbols) to $T_{\text {eff }}=T^{0.7} T_{V}^{0.3}$ (filled symbols) has almost no effect. While thermal nonequilibrium is present in the shock layer there is not enough thermal nonequilibrium over substantial portions of the shock layer to make a significant effect on aerodynamics through effective kinetic rates.

The sensitivity of $C_{A}(2)$ and $C_{m}(2)$ with respect to $\rho_{\infty}$ and kinetic model at point $\mathrm{C}$ are shown respectively in Figs. 14 and 15. Aerodynamic coefficients are still insensitive to perturbations as large as $40 \%$ about the nominal density of $1.1310^{-3} \mathrm{~kg} / \mathrm{m}^{3}$. In contrast, the effect on $C_{m}(2)$ of a $7 \%$ perturbation in velocity at the same density is large, as observed by comparing point B in Table 1 with the result for $\rho_{\infty}$ equal to .001 $\mathrm{kg} / \mathrm{m}^{3}$ in Fig. 14. The effects of dissociation rate constant for $\mathrm{CO}_{2}$ on aerodynamics is stronger at point $\mathrm{C}$ than at Point A. Previous characterization of the flow at this point as "near equilibrium" over-simplified the role of gas kinetics during this portion of the trajectory. Doubling the rate factor moves $C_{m}(2)$ closer to a predicted domain of static stability. Reducing the rate by a factor of 10 moves $C_{m}(2)$ closer to a predicted domain of static instability. Nevertheless, the change in $C_{A}(2)$ over this entire range of kinetic model adjustments is less than $0.6 \%$.

The movement of the sonic line associated with changes in the $\mathrm{CO}_{2}$ dissociation rate at Point $\mathrm{C}$ are shown in Fig. 16. The baseline kinetic model results are in Fig. 16 c, with rate factor equal to 1 . Increasing rate factor causes the sonic bubble to shrink and

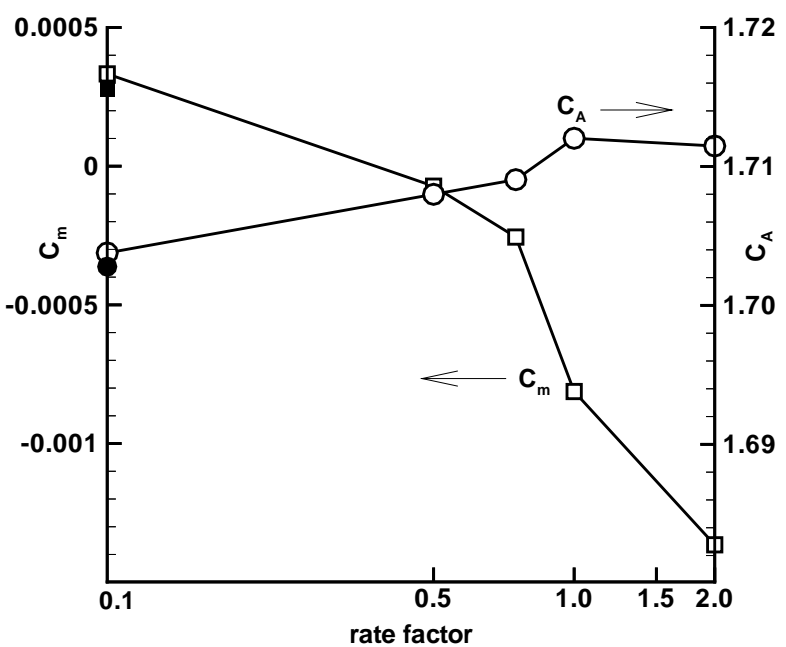

Fig. 15 The computed sensitivity of Pathfinder aerodynamic coefficients on dissociation rate coefficient for $\mathrm{CO}_{2}$ at a freestream density $\mathbf{0 . 0 0 1 1 2 7}$ $\mathrm{kg} / \mathrm{m}^{3}$ and velocity of $3996 . \mathrm{km} / \mathrm{s}$.

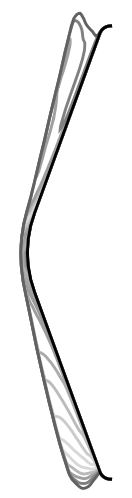

(a) 0.1

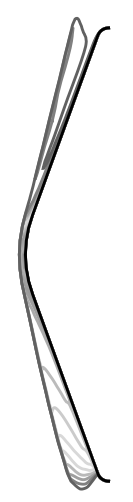

(b) 0.5

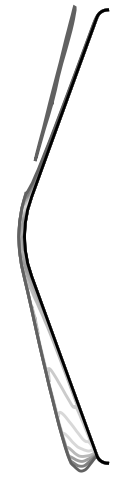

(c) 1.0

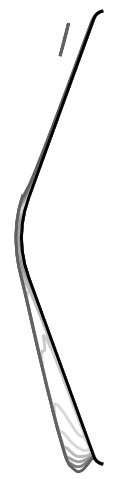

(d) 2.0
Fig. 16 The computed sensitivity of Pathfinder sonic line location on dissociation rate coefficient for $\mathrm{CO}_{2}$ at a freestream density $0.001127 \mathrm{~kg} / \mathrm{m}^{3}$ and velocity of $3996 . \mathrm{km} / \mathrm{s}$.

the moment coefficient to move into the domain of static stability. Decreasing rate factor causes the sonic bubble to grow and approach a condition of static instability. The sonic bubble emanates from an inflection point in the bow shock which eventually disappears with the merging of the bubble with the sonic line on the edge of the boundary layer.

The sensitivity tests at Points $\mathrm{A}$ and $\mathrm{C}$ show that aerodynamic database is insensitive to perturbations in density as large as $10 \%$. It is believed this lack of sensitivity would persist across the entire hypersonic, continuum domain for the Pathfinder vehicle shape. 
The sensitivity will manifest itself during the transitional flow regime prior to continuum; in fact, Moss et. al. ${ }^{11}$ predicted static instabilities in the transitional regime for Pathfinder as a function of Knudsen number variation.

The baseline thermochemical model yields values for $C_{m}(2)$ which are consistent, within estimated uncertainty bands, of the measured normal accelerations. Point B shows the greatest disagreement between measurement and simulation of normal accelerations. Agreement would likely be improved using a simulation with a larger offset $\mathrm{CG}$ and/or slower $\mathrm{CO}_{2}$ dissociation rates to define $C_{m}$.

The normal acceleration data is useful as a consistency check for the simulation models, much like measurements of shock standoff distance in ground based experiments are used. While one could not use the data by itself to propose changes to any element(s) of the chemical kinetic model, one should question any model changes that cause inconsistent values of $C_{m}(2)$ with measured normal accelerations along any point on the Pathfinder trajectory. In the tests conducted herein, rather large perturbations in the kinetic model are required before computed values for $C_{m}(2)$ are pushed outside of the expected limits based on measured normal accelerations.

\section{Conclusions}

(1) Predictions of two regions of static instability during the hypersonic, continuum portion of the Mars Pathfinder trajectory are verified by flight measurements of normal accelerations. The relation of these instabilities to sonic line movement between the nose and the shoulder as a function of trajectory point and kinetic model is demonstrated.

(2) Perturbations to density as large as $10 \%$ about nominal values for the reconstructed trajectory during the hypersonic, continuum regime cause negligible change (less than $1 \%$ ) in $C_{A}$. Large changes in the kinetic model in this regime cause changes in $C_{A}$ of approximately $1 \%$. Given other sources of uncertainty unrelated to the simulation model, the reconstructed density, with uncertainty linearly related to uncertainty in $C_{A}$, is expected to have approximately $4 \%$ uncertainty in the hypersonic, continuum regime.

(3) The occurrence of static instabilities as recorded by normal accelerations during the Mars Pathfinder entry, descent, and landing (EDL) provide an opportunity for computational fluid dynamic (CFD) code validation. Computed moment coefficients are consistent with identified ranges for measured normal accelerations indicating flight in a statically stable or unstable regime. The definitions of these ranges for $C_{m}$ are independent of CFD simulation; they are extracted from 6 degree-of-freedom trajectory simulations using several aerodynamic coefficient models for flight through the Mars atmosphere. The present simula- tions are consistent with measured data within the defined ranges, including the uncertainty band.

\section{Acknowledgements}

The authors thank Robert Blanchard of the Aerothermodynamics Branch, Langley Research Center for supplying data for the reconstructed atmosphere, trajectories, and accelerometers.

\section{References}

${ }^{1}$ Gnoffo, P. A., Weilmuenster, K. J., Braun, R. D., and Cruz, C. I., "Influence of Sonic-Line Location on Mars Pathfinder Probe Aerothermodynamics," Journal of Spacecraft and Rockets, Vol. 33, No. 2, March-April 1996, pp. 169-177.

${ }^{2}$ Braun, R. D., Powell, R. W., Cruz, C. I., Gnoffo, P. A., and Weilmuenster, K. J., "Six Degree-of-Freedom Atmospheric Entry Analysis for the Mars Pathfinder Mission," AIAA 950456, January 1995.

${ }^{3}$ Brauer, G. L., Cornick, D. E., and Stevenson, R., "Capabilities and Applications of the Program to Optimize Simulated Trajectories (POST)," NASA CR 2770, February 1977.

${ }^{4}$ Spencer, D. A., Thurman, S. W., Peng, C.-Y., Blanchard, R. C., and Braun, R. D., "Mars Pathfinder Atmospheric Entry Reconstruction," AAS 98-146, AAS/AIAA, February 1998.

${ }^{5}$ Gnoffo, P. A., Weilmuenster, K. J., H. Harris Hamilton, I., Olynick, D. R., and Venkatapathy, E., "Computational Aerothermodynamic Design Issues for Hypersonic Vehicles," AIAA 97-2473, AIAA, June 1997.

${ }^{6}$ Intrieri, P. F., Rose, C. E. D., and Kirk, D. B., "Flight Characteristics of Probes in the Atmospheres of Mars, Venus and the Outer Planets," Acta Astronautica, Vol. 4, 1977, pp. 789-799.

${ }^{7}$ Justus, C. G., James, B. F., and Johnson, D. L., "Mars Global Reference Atmospheric Model (Mars-GRAM 3.3.4): Programmers Guide," TM 108509, NASA, May 1996.

${ }^{8}$ Clancy, R., Lee, S., Gladstone, G., McMillan, W., and Rousch, T., "A New Model of Mars Atmospheric Dust Based on Analysis of Ultraviolet Through Infrared Observations from Mariner-9, Viking, and Phobos," Journal of Geophysical Research, Vol. 100, 1995, pp. 5251-5263.

${ }^{9}$ Spencer, D. and Braun, R., "Mars Pathfinder Atmospheric Entry: Trajectory Design and Dispersion Analysis," Journal of Spacecraft and Rockets, Vol. 33, No. 5, Sept.-Oct 1996, pp. 670676.

${ }^{10}$ Mitcheltree, R. A., "Aerothermodynamic Methods for a Mars Environmental Survey Mars Entry," Journal of Spacecraft and Rockets, Vol. 31, No. 3, May-June 1994, pp. 516-523.

${ }^{11}$ Moss, J. N., Wilmoth, R. G., and Price, J. M., "DSMC Simulations of Blunt Body Flows For Mars Entries: Mars Pathfinder and Mars Microprobe Capsules," AIAA 97-2508, June 1997. 